\title{
A Brief Note on the Clock-Hypothesis
}

\author{
Andreas Schlatter \\ Emeran AG, Küttigen, Switzerland \\ Email: schlatter.a@bluewin.ch
}

How to cite this paper: Schlatter, A. (2016) A Brief Note on the Clock-Hypothesis. Journal of Modern Physics, 7, 2098-2102. http://dx.doi.org/10.4236/jmp.2016.715183

Received: October 26, 2016

Accepted: November 7, 2016

Published: November 10, 2016

Copyright $\odot 2016$ by author and Scientific Research Publishing Inc. This work is licensed under the Creative Commons Attribution International License (CC BY 4.0).

http://creativecommons.org/licenses/by/4.0/ (c) (†) Open Access

\begin{abstract}
The clock-hypothesis is the fundamental assumption in the theories of relativity that duration, measured by clocks, is proportionate to the length of their respective world lines. Over the years, there have been contributions both, theoretical and experimental in nature, either confirming or questioning this hypothesis. We give an elementary model of two classes of clocks, which turn out to be relativistic clocks, and by doing so also offer a basis to see the limitations of the clock-hypothesis. At the same time, we find support for a hypothesis of L. de Broglie, regarding the existence of an internal clock of electrons. Our aim is to give a precise, yet accessible account of the subject.
\end{abstract}

\section{Keywords}

Quantum Physics, Special Relativity, Schroedinger Evolution, de Broglie Internal Clock

\section{Introduction}

In his seminal work of 1905 [1] and again in a later paper [2] in 1907 A, Einstein originated the idea that clocks actually measure a duration, which is proportionate to the length of their respective world-lines ${ }^{1}$; an assumption, which is known today as the clock-hypothesis. This hypothesis underlies much of the geometric structure of the theories of relativity [3]. The clocks in the original work are no further specified other than they are ideal, point-like devices. Much work has been done since then on the topic of relativistic clocks, either by experimentally confirming the hypothesis through detection of time-dilation in specific clock-devices [4] [5] [6] [7], or by showing limitations of the hypothesis including critique on the before mentioned experiments [8] [9] [10]. Experiments have so far mainly been based on either moving particles, like muons, or on atomic clocks, i.e. atoms emitting/absorbing light without recoil, possible due to

${ }^{1}$ The notionoffour dimensional worldline was introducedonlylater in 1908 by H. Minkowski. 
the Mössbauer-effect [11]. Some recent work focuses on the fact that real clocks are never point-like entities and that consequently forces between its constituent parts or between different clock-devices can influence the duration-measurement at the quantum level. The considerable machinery of quantum-field theory in curved space-time is applied in [9] to describe gravitational effects on the clocks or in [10] to find e.g. an impact of particle-creation on the duration measurement. Other recent work centers around the question, which clock-devices qualify as relativistic clocks and which ones do not [12].

In this short note, we will use a simple model, using elementary tools only, to describe two classes of clocks: moving particle-based clocks, especially electrons, and atomic clocks. We will show that they are indeed relativistic clocks and will rediscover a hypothesis by L. de Broglie [13], concerning internal clocks of electrons. At the same time, we will be able to understand some of the critical arguments mentioned above. Our aim is to give a precise, yet accessible account of the subject.

\section{Relativistic Clocks}

Let's first consider a quantum system represented by a wave function $\psi$ determined by the Schrödinger equation

$$
\begin{aligned}
& i \hbar \partial_{t} \psi=H \psi \\
& \psi(0)=\psi_{0} .
\end{aligned}
$$

By the time-energy inequality and a result in [14], there holds for the minimal time $T$ until $\psi_{0}$ evolves into an orthogonal state $\psi_{T},\left\langle\psi_{T}, \psi_{0}\right\rangle=0$,

$$
T=\max \{h / 4 E, h / 4 \Delta E\} .
$$

In (2) $E$ and $\Delta E$ represent the first and second moment of the energy operator $H$. Let us assume that the system $\psi$ is in its local rest-frame and ticks with frequency $T^{2}$. By (2) it produces in an incremental time-step $\mathrm{d} t$ the (incremental) number of flips (orthogonal states) $\mathrm{d} \aleph$

$$
\mathrm{d} \aleph(t)=\mathrm{d} t / T .
$$

To describe the first class of clocks based on (3) we think of the system $\psi$ to be a particle with total (average) relativistic energy $E$ and describe it in a semi-classical way. Then by (2) Equation (3) turns in the local rest frame into

$$
\mathrm{d} \aleph(t)=(4 E / h) \mathrm{d} t .
$$

The energy $E$ represents kinetic and inner energy, defining the time-component of the four momentum $p(t)=(E(t) / c, p(t))$ along the world-line $x(t)=(c t, x(t))$. We can parametrise $x$ by its write four-length $s$ and (4) in covariant form by

$$
\mathrm{d} \aleph(s)=(4 / h)\langle p(s), \mathrm{d} x(s)\rangle_{L} .
$$

The expression $\langle\cdot \mid \cdot\rangle_{L}$ denotes the four-scalar product. We will omit the subscript in the sequel. We can now write with $m_{0} \neq 0$ denoting the rest mass of the particle

${ }^{2}$ We need, of course, an assumption on size to justify a singel time-parameter $t$ and hence (1). 


$$
\mathrm{d} \aleph(s)=(4 / h)\langle p, \mathrm{~d} x / \mathrm{d} s\rangle \mathrm{d} s=\left(4 / m_{0} h\right)\langle p, p\rangle \mathrm{d} s .
$$

There holds along any (time-like) world line

$$
\langle p, p\rangle=m_{0}^{2} c^{2} .
$$

For electrons, satisfying $i \hbar \gamma^{\mu} \partial_{\mu} \psi=m_{0} c \psi, 0 \leq \mu \leq 4$, where $i \hbar \gamma^{\mu} \partial_{\mu}$ denotes the Dirac four-momentum operator, Equation (7) holds indeed on the expectation-value level.

Therefore, by integration, we get for any distance $\tau$ on the world line

$$
\aleph(\tau)=4\left(m_{0} c^{2} / h\right) \tau \text {. }
$$

Equation (8) confirms that electrons can indeed act as relativistic clocks, ticking with an internal frequency of a multiple of $m_{0} c^{2} / h$. This is an idea, which was already suggested by L. de Broglie [13]. It is interesting to note that, if we chose the minimal time to pass through $N$ orthogonal states, $N$ large, instead of the minimal time to flip between two states, then we get $T=h / 2 E \quad[14]$ and hence instead of (8)

$$
\aleph(\tau)=2\left(m_{0} c^{2} / h\right) \tau,
$$

a result, which fits well in the discussion in e.g. [15].

Another class of clocks consists of atomic devices, where $\Delta E=h v_{0}$ is the energy difference between two quantum levels $|1\rangle$ and $|0\rangle$ of a photon emitting/absorbing atom with Hamiltonian $H=E_{1}|1\rangle\left\langle 1\left|+E_{0}\right| 0\right\rangle\langle 0|$, which can be recoil-free due to the Mössbauer effect $[11]^{3}$. By (2) and (3) we get in the local rest-frame

$$
\mathrm{d} \aleph(t)=(4 \Delta E / h) \mathrm{d} t=4 v_{0} \mathrm{~d} t .
$$

The most direct ansatz for a covariant formulation of (10) is to simply chose the length $s$ of the world-line of the clock as the parameter. We get

$$
\mathrm{d} \aleph(s)=4 v_{0} \mathrm{~d} s .
$$

If (11) is correct, then atomic clocks are indeed relativistic clocks as well. Let's gather evidence for it and assume first that the clock (atom) is in transversal uniform motion relative to an observer at rest. We have

$$
4 v_{0} \mathrm{~d} s=4 v_{0}(\mathrm{~d} t / \gamma)=4 v \mathrm{~d} t
$$

Therefore, we get for the frequency $v$ in the frame of the observer

$$
v=v_{0} / \gamma,
$$

where as usual $\gamma=1 / \sqrt{1-v^{2} / c^{2}}$. This is the (transversal) Doppler-effect and its presence in atomic clocks has been observed in experiments [5].

With $T_{0}=1 / 4 v_{0}$ denoting the oscillation-period, Equation (11) also leads to the expression for the time-dilation (red-shift) of a clock at rest in a static space-time, relative, for example, to an observer far away from the source of the gravitational field. With the line-element being $\mathrm{d} s=\sqrt{g_{00}} \mathrm{~d} t$, we have

$$
\mathrm{d} s / T_{0}=\sqrt{g_{00}} \mathrm{~d} t / T_{0}=\mathrm{d} t / T .
$$

\footnotetext{
${ }^{3}$ The expectation value is taken with respect to the state $1 / \sqrt{2}(|1\rangle+|0\rangle)$.
} 
Hence

$$
T=T_{0} / \sqrt{g_{00}}
$$

The effect of gravitational red-shift on atomic clocks (15) has indeed been observed as well [6]. Expressions (13) and (14) and their empirical verification are evidence that (11) is correct.

\section{Some Observations}

Experiments with the two kinds of clocks support the clock-hypothesis and our elementary model gives an accessible theoretical framework to explain it. The basis was that we managed to establish a relation (3) between the internal evolution of a system and the length of the world line it is supposed to pass through. While, of course, we compromised in principle by choosing a semi-classical treatment, it seems that, compared to moving particles, atomic clocks are even less ideal devices though, since they are not point-like, and Equation (11) only holds neglecting any real clock components other than the photon-emission/absorption. There might be effects on the photon by other parts of the clocks [9] or a more sophisticated description of the oscillator by a quantum-field might lead to particle-creation [10]. Qualitatively, we can see already from our model, e.g. by (4) or (10), that particle-creation must lead to a time-dilation in the order of magnitude of the energy of the created particles, which is no longer available to process the clock.

It is not obvious to see how systems, which cannot be brought into a covariant form of type (3)

$$
\mathrm{d} \aleph(s) / \mathrm{d} s=1 / T=\text { const. }
$$

could support the clock-hypothesis. Special relativity is the result of a conceptual merger of (classical) particle dynamics with electrodynamics. It is interesting to notice, that the clock-hypothesis is indeed best confirmed by devices, which are based on either of the two pillars: particle motion or electromagnetism. Systems, whose internal evolution base on other forces, like thermal energy, seem more resistant to confirm the hypothesis [12].

More work will be done on the question of clocks in order to tackle the deeper issue, namely the one of the true nature of time.

\section{References}

[1] Einstein, A. (1905) Annalen der Physik, 17, 891-921. http://dx.doi.org/10.1002/andp.19053221004

[2] Einstein, A. (1907) Jahrbuch der Radioaktivität, 411-462.

[3] Maudlin, T. (2012) Philosophy of Physics, Volume 1: "Space and Time". Princeton University Press, Princeton.

[4] Rossi, B. and Hall, D.B. (1941) Physical Review, 59, 223-228. http://dx.doi.org/10.1103/PhysRev.59.223

[5] Ives, H.E. and Stilwell, G. (1938) Journal of the Optical Society of America, 28, 215. 
http://dx.doi.org/10.1364/JOSA.28.000215

[6] Pound, R.V. and Rebka, G.A. (1959) Physical Review Letters, 3, 439-441. http://dx.doi.org/10.1103/PhysRevLett.3.439

[7] Hafele, J. and Keating, R. (1972) Science, 177, 168-170. http://dx.doi.org/10.1126/science.177.4044.168

[8] Bonnizoni, I. and Giuliani, G. (2000) The Interpretations by Experimenters of Experiments on “Time Dilation": 1940-1970 ca. arXiv:physics/0008012 [physics.hist-ph].

[9] Zych, M., Costa, F.M., Pikovski, I., Brukner, C. and Ralph, T.C. (2016) Journal of Physics. Conference Series, 723. http://dx.doi.org/10.1088/1742-6596/723/1/012044

[10] Ahmadi, M., Bruschi, D.E., Sabin, C., Adesso, G. and Fuentes, I. (2014) Scientific Reports, 4, Article ID: 4996. http://dx.doi.org/10.1038/srep04996

[11] Mössbauer, R. (1958) Zeitschriftfür Physik, 151, 124-143.

[12] Borghi, C. (2016) Foundations of Physics, 46, 1374-1379. http://dx.doi.org/10.1007/s10701-016-0030-y

[13] De Broglie, L. (1924) Annales de Physique, 3, 22.

[14] Margolus, N. and Levitin, L.B. (1998) Physica D, 120, 188-195. http://dx.doi.org/10.1016/S0167-2789(98)00054-2

[15] Osche, G.R. (2011) Annales de la Fondation Louis de Broglie, 36, 61-71.

\section{Submit or recommend next manuscript to SCIRP and we will provide best service} for you:

Accepting pre-submission inquiries through Email, Facebook, LinkedIn, Twitter, etc. A wide selection of journals (inclusive of 9 subjects, more than 200 journals)

Providing 24-hour high-quality service

User-friendly online submission system

Fair and swift peer-review system

Efficient typesetting and proofreading procedure

Display of the result of downloads and visits, as well as the number of cited articles

Maximum dissemination of your research work

Submit your manuscript at: http://papersubmission.scirp.org/

Or contact jmp@scirp.org 